\title{
Pynamical: Model and visualize discrete nonlinear dynamical systems, chaos, and fractals
}

\section{DOI: $10.21105 /$ jose. 00015}

\section{Software}

- Review ct

- Repository ${ }^{\top}$

- Archive ct

Submitted: 15 May 2018

Published: 21 June 2018

\section{License}

Authors of papers retain copyright and release the work under a Creative Commons Attribution 4.0 International License (CC-BY).

\section{Geoff Boeing $^{1}$}

1 University of California, Berkeley

\section{Summary}

Pynamical is an educational Python package for introducing the modeling, simulation, and visualization of discrete nonlinear dynamical systems and chaos, focusing on onedimensional maps (such as the logistic map and the cubic map). Pynamical facilitates defining discrete one-dimensional nonlinear models as Python functions with just-in-time compilation for fast simulation. It comes packaged with the logistic map, the Singer map, and the cubic map predefined. The models may be run with a range of parameter values over a set of time steps, and the resulting numerical output is returned as a pandas DataFrame. Pynamical can then visualize this output in various ways, including with bifurcation diagrams (May 1976), two-dimensional phase diagrams (Packard et al. 1980), three-dimensional phase diagrams, and cobweb plots (Hofstadter 1985). These visualizations enable simple qualitative assessments of system behavior including phase transitions (Feigenbaum 1983), bifurcation points (Sander and Yorke 2015), attractors and limit cycles (Grebogi, Ott, and Yorke 1987), basins of attraction (Sprott and Xiong 2015), and fractals (Mandelbrot 1967, 1983).

Although most real-world systems are nonlinear dynamical systems, their mathematical analysis is notoriously difficult because they cannot be simply broken down into individual parts then recombined linearly (Strogatz 2014). Instead, researchers have long relied on visualization techniques to make system behavior comprehensible (Alpigini 2004; Layek 2015). Such visualization is useful for exploring nonlinear time series data (Bradley and Kantz 2015; Boeing 2016). Pynamical facilitates the modeling, visualization, and exploration of this rich nonlinear behavior, as demonstrated in Figures 1 and 2. Accordingly this package has various applications in research, engineering, and particularly pedagogy. For instance, it allows students to explore and model any well-defined discrete nonlinear dynamical system (with just one line of code) and then simulate its behavior over $n$ generations in time (with just one more line of code). This facilitates easy experimentation in parameter space and sensitivity analysis. Moreover, Pynamical easily produces publication-quality visualizations to illustrate findings without having to reinvent the wheel with ad hoc algorithms to produce, reshape, and plot simulation data. Finally, it enables the animation of such systems, to explore and present their qualitative behavior and evolution.

The latest release of the software can be installed via conda or pip and full documentation can be found at https://pynamical.readthedocs.io.

\section{Statement of Need}

These visualization methods are useful for introducing nonlinearity to students, and Pynamical fills a current need: the existing software landscape typically requires that such 
tools either a) be developed ad hoc from scratch or b) exist as add-ons to expensive closedsource commercial packages like MATLAB (Tomida 2008). Pynamical addresses the need for a fast, simple, extensible, free, and open-source Python package for the analysis of such systems in natural and social science education and research. It depends on the pandas, numpy, numba, and matplotlib Python packages for fast simulation and attractive visualizations to explore system behavior. This software was developed for two purposes: 1) as a pedagogy tool and 2) to produce figures, diagrams, and animations. It has been used in the classroom to introduce students to the basics of nonlinearity, unpredictable system behavior, simple parameter space exploration, and the geometric logic of fractals. This has proven particularly useful for students outside the traditional domains of mathematics and physics to approach these topics that are also relevant to the social sciences, such as modeling and understanding systems and patterns in geography and urban planning. As it focuses primarily on one-dimensional maps, this software could be most useful at the beginning of an introductory course on dynamical systems (e.g., following Strogatz's textbook (Strogatz 2014)). It can also be used to generate publication-quality figures for lessons and tutorials, as well as animated visualizations of system behavior and patterns.

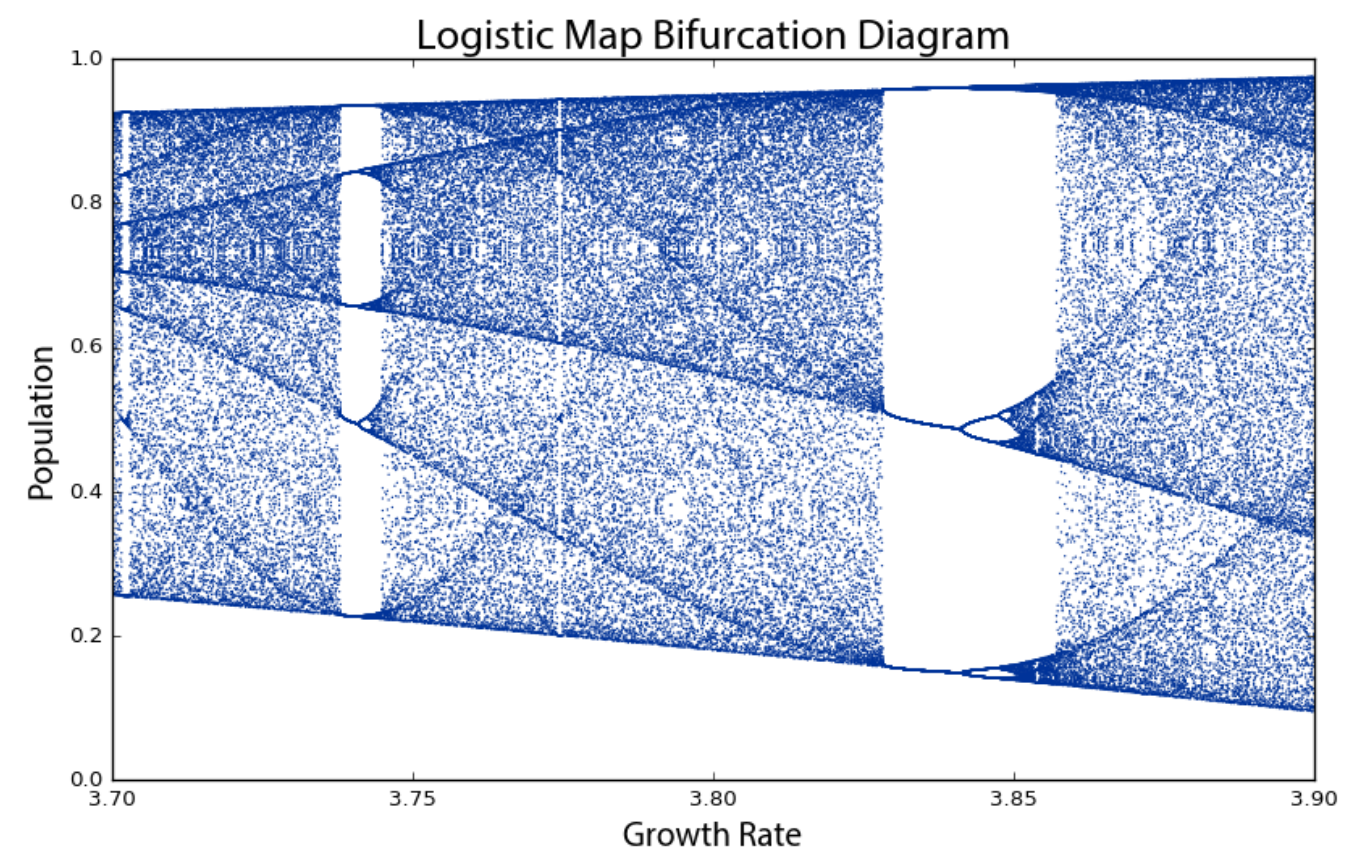

Figure 1. Bifurcation diagram of the logistic map's chaotic regime.

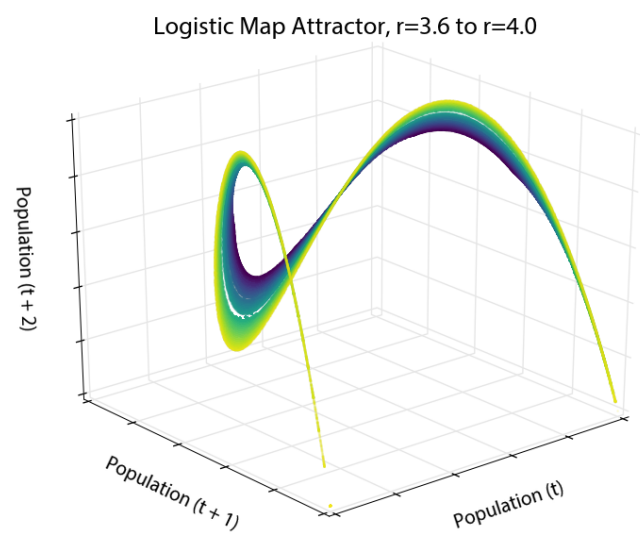

Logistic Map Attractor, $r=3.6$ to $r=4.0$

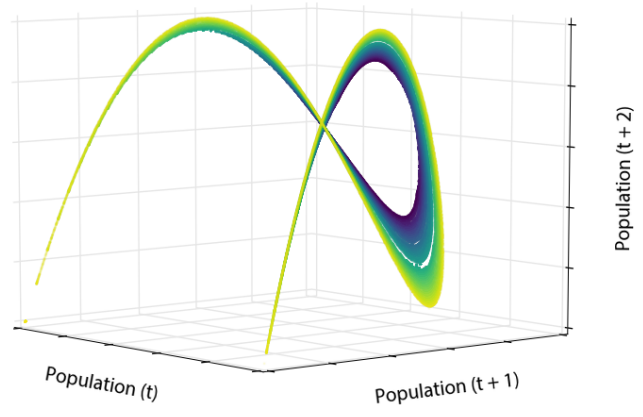

Figure 2. Phase diagram of the logistic map's chaotic regime. 


\section{References}

Alpigini, James J. 2004. "Dynamical System Visualization and Analysis via Performance Maps." Information Visualization 3 (4):271-87. https://doi.org/10.1057/palgrave.ivs. 9500082.

Boeing, Geoff. 2016. "Visual Analysis of Nonlinear Dynamical Systems: Chaos, Fractals, Self-Similarity and the Limits of Prediction." Systems 4 (4):37. https://doi.org/10.3390/ systems4040037.

Bradley, Elizabeth, and Holger Kantz. 2015. "Nonlinear Time-Series Analysis Revisited." Chaos: An Interdisciplinary Journal of Nonlinear Science 25 (9):097610. https://doi.org/ 10.1063/1.4917289.

Feigenbaum, Mitchell J. 1983. "Universal Behavior in Nonlinear Systems." Physica D: Nonlinear Phenomena 7 (1-3):16-39. https://doi.org/10.1016/0167-2789(83)90112-4.

Grebogi, Celso, Edward Ott, and James A. Yorke. 1987. "Chaos, Strange Attractors, and Fractal Basin Boundaries in Nonlinear Dynamics." Science 238 (4827):632-38. https: //doi.org/10.1126/science.238.4827.632.

Hofstadter, Douglas R. 1985. "Mathematical Chaos and Strange Attractors." In Metamagical Themas: Questing for the Essence of Mind and Pattern, 364-95. New York, NY: Basic Books.

Layek, G. C. 2015. An Introduction to Dynamical Systems and Chaos. New Delhi, India: Springer India.

Mandelbrot, Benoit B. 1967. "How Long Is the Coast of Britain? Statistical SelfSimilarity and Fractional Dimension." Science 156 (3775):636-38. https://doi.org/10. 1126/science.156.3775.636.

- 1983. The Fractal Geometry of Nature. New York, NY: Macmillan.

May, Robert M. 1976. "Simple Mathematical Models with Very Complicated Dynamics." Nature 261 (5560):459-67. https://doi.org/10.1038/261459a0.

Packard, N. H., J. P. Crutchfield, J. D. Farmer, and R. S. Shaw. 1980. "Geometry from a Time Series." Physical Review Letters 45 (9):712-16. https://doi.org/10.1103/ PhysRevLett.45.712.

Sander, Evelyn, and James A. Yorke. 2015. "The Many Facets of Chaos." International Journal of Bifurcation and Chaos 25 (4):1530011. https://doi.org/10.1142/ S0218127415300116.

Sprott, J. C., and Anda Xiong. 2015. "Classifying and Quantifying Basins of Attraction." Chaos: An Interdisciplinary Journal of Nonlinear Science 25 (8):083101. https://doi.org/ $10.1063 / 1.4927643$.

Strogatz, Steven H. 2014. Nonlinear Dynamics and Chaos. 2nd ed. Boulder, CO: Westview Press.

Tomida, Akemi Galvez. 2008. "Matlab Toolbox and GUI for Analyzing One-Dimensional Chaotic Maps." In, 321-30. Perugia, Italy: IEEE. https://doi.org/10.1109/ICCSA.2008. 7. 